\title{
Short communication: Prevalence of Staphylococcus aureus and methicillin- resistant $S$. aureus in bulk tank milk from dairy goat farms in Northern Italy
}

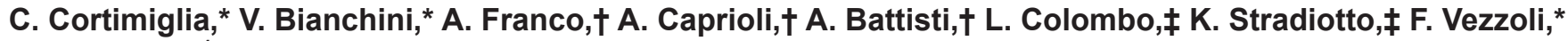 \\ and M. Luini ${ }^{* 1}$ \\ *Istituto Zooprofilattico Sperimentale della Lombardia e dell'Emilia Romagna, Lodi 26900, Italy \\ †Istituto Zooprofilattico Sperimentale delle Regioni Lazio e Toscana, Rome 00178, Italy \\ $\ddagger$ Associazione Regionale Allevatori, Crema 26013 (CR), Italy
}

\section{ABSTRACT}

Staphylococcus aureus is regarded as a leading cause of mastitis in goats. However, few data are available on the presence of methicillin-resistant $S$. aureus (MRSA) in this species. The aim of this study was to assess the prevalence of $S$. aureus and MRSA in bulk tank milk samples from dairy goat farms in Northern Italy. Eighty-five out of 197 samples (43.1\%) tested positive for $S$. aureus with counts ranging from 10 to more than $1.5 \times 10^{4} \mathrm{cfu} / \mathrm{mL}$. The MRSA was screened by both direct plating followed by a disk diffusion test to evaluate methicillin resistance and a selective enrichment method. Methicillin-resistance was confirmed by $m e c A$-specific PCR. Methicillin-resistant S. aureus was identified in 4 samples (2.0\%) and multilocus sequence typing (MLST) showed the presence of livestock-associated MRSA belonging to lineages ST398 $(\mathrm{n}=3)$ and ST1 $(n=1)$. In one case we demonstrated that the same MRSA strain was able to persist over time on the farm, being isolated from both bulk tank milk and the udder of 3 goats 1 yr after the first isolation. The high prevalence of $S$. aureus-positive herds detected in this study and the presence of MRSA strains belonging to livestock-associated genotypes is of concern, and represents a novel finding in the Italian dairy goat production system. The application of stringent measures for the control of $S$. aureus mastitis at the farm level seems appropriate to reduce the economic losses, and to minimize the risk of foodborne illness and the transmission of MRSA to humans by occupational exposure.

Key words: Staphylococcus aureus, methicillin-resistance, dairy goat, bulk tank milk

Received October 1, 2014

Accepted December 17, 2014.

${ }^{1}$ Corresponding author: mariovittorio.luini@izsler.it

\section{Short Communication}

Staphylococcus aureus is considered a major pathogen in dairy goats and is responsible for both clinical and subclinical intramammary infections (Bergonier et al., 2003). The prevalence of $S$. aureus positive herds, estimated by bulk tank milk analysis, ranges from 16.7 to $96.2 \%$ in different countries, including Italy (Muehlherr et al., 2003; Jørgensen et al., 2005; Chu et al., 2012; Spanu et al., 2013). Staphylococcus aureus intramammary infections result in significant economic losses due to reduced milk production and poor milk quality, and represent a source of contamination of raw milk and dairy products.

The epidemiology of $S$. aureus in animals has gained increasing attention due to the emergence of livestockassociated methicillin-resistant strains (LA-MRSA; Guardabassi et al., 2013). High-level resistance to $\beta$-lactam antimicrobials in $S$. aureus strains is conferred by the mecA gene, which encodes a modified penicillin binding protein. It was first observed in the early 1960s and rapidly spread through hospital-associated clones, mostly in nosocomial settings. In the late 1990s, new MRSA clones emerged in the community, being increasingly reported in healthy individuals without history of hospitalization (Guardabassi et al., 2013). In the last few years, MRSA has been identified with increased frequency in different domestic animals, primarily pigs and cattle in the European Union, including Italy (Battisti et al., 2010; Feltrin et al., 2013). In Italy, LA-MRSA from food producing animals belongs predominantly to clonal complex (CC) 398, although other clones, such as CC97 and CC1, are also widespread (Battisti et al., 2010; Franco et al., 2011; Feltrin et al., 2013). Consequently, MRSA-carrier animals and farm environments can be responsible for the exposure of farmers and veterinarians to the pathogen (Pantosti, 2012), and cause clinical disease (Pan et al., 2009).

Limited data on the presence of MRSA in dairy goats are available (Stastkova et al., 2009; Aras et al., 2012; Chu et al., 2012), and in Italy both Virdis et al. (2010) 
and Foti et al. (2012) previously failed to detect MRSA in goat milk.

Staphylococcus aureus is also involved in foodborne disease cases resulting from the contamination of food by preformed enterotoxins. Dairy products, especially fresh cheeses, could be sources of such human intoxication (Kadariya et al., 2014). Conversely, the isolation of MRSA from dairy products is relatively rare, with the exception of raw milk and fresh cheeses made from contaminated milk (Normanno et al., 2007; Doyle et al., 2012) and so far major LA-MRSA clones have been considered of minor significance as a cause of food poisoning in the European Union (EFSA, 2009).

Having considered the implications of $S$. aureus in contagious mastitis and the risk of MRSA transmission for occupationally exposed people, the study was aimed at estimating the prevalence of $S$. aureus and MRSA in bulk tank milk of dairy goat herds located in the Lombardy Region (Northern Italy), as a preliminary step for risk assessment purposes.

Bulk tank milk samples intended for quality controls scheduled by the regional breeding association (ARAL, Associazione Regionale Allevatori della Lombardia, Crema, Italy) from 197 different dairy goat farms were collected between July and October 2012. The number of farms investigated represented $2.5 \%$ of the Lombardy dairy goat herds, corresponding to $11.3 \%$ of the total reared goats and accounting for $22.7 \%$ of the produced milk. In these farms the average herd size was 42 lactating goats, with an average production of $540 \mathrm{~kg}$ of milk per lactation.

The following year, during the subsequent lactation (summer 2013), bulk tank milk samples from MRSApositive farms detected the previous year were tested again. At the same time, in 2 of these farms (indicated as farm A and B), milk samples from both udder halves of all lactating goats $(\mathrm{n}=237$ and $\mathrm{n}=102$, respectively) were also collected.

After collection, milk samples were transported chilled to the laboratory and stored at $-20^{\circ} \mathrm{C}$ until bacteriological analysis because previous studies showed that freezing has no effect on the viability of $S$. aureus (Jørgensen et al., 2005). Samples were diluted 1:10 in $0.1 \%$ sterile peptone water and $100 \mu \mathrm{L}$ of the milk and of the decimal dilution were spread on blood agar (BA) supplemented with 5\% defibrinated sheep blood and on Baird Parker agar supplemented with rabbit plasma fibrinogen (BP-RPF; Oxoid Ltd., Basingstoke, UK). The plates were incubated at $37^{\circ} \mathrm{C}$ for $48 \mathrm{~h}$ and analyzed after 24 and $48 \mathrm{~h}$. Colonies developing a typical coagulase halo on BP-RPF agar were considered suspected $S$. aureus, as were hemolytic colonies on BA testing positive in a tube coagulase test (Coagulase plasma-EDTA, Biolife srl, Milan, Italy). Up to 4 typical colonies on BA or BP-RPF from each positive sample were screened for methicillin susceptibility by disk diffusion test with $1 \mu \mathrm{g}$ of oxacillin on Mueller-Hinton Agar plates (both from Oxoid Ltd.), following the CLSI M31-A3 Standard in use at that time (CLSI, 2008). Isolates with inhibition zone diameters $<13 \mathrm{~mm}$ (i.e., intermediate or resistant according to M31-A3 CLSI clinical breakpoints) were further confirmed by multiplex PCR targeting the $S$. aureus-specific nuc gene (Cremonesi et al., 2005) and the mecA gene (Zhang et al., 2005) according to the method reported by Benedetti et al. (2010). Retrospectively, in 2014, all isolates that showed oxacillin inhibition zone diameters $\leq 21$ $\mathrm{mm}$ ( $\mathrm{n}=62$, from 20 bulk milk samples) were further screened for mecC by PCR, following the method described by Cuny et al. (2011), because more recent work (Skov et al., 2014) demonstrated that the disk diffusion oxacillin test may fail to detect a proportion of $m e c C$-positive isolates, including those classified as intermediate or even susceptible according to the CLSI M31-A3 Standard.

The number of colony-forming units per milliliter for S. aureus was calculated as the weighted average of the number of typical colonies on the 2 BP-RPF plates. To estimate colony-forming units per milliliter for MRSA, we first counted the number of screened colonies confirmed as $m e c A$-positive out of the 4 colonies tested and then we applied the proportion with respect to the total number of $S$. aureus colonies.

In addition to direct plating, $3 \mathrm{~mL}$ of milk was enriched in Mueller-Hinton broth (Oxoid Ltd.) with 7.5\% $\mathrm{NaCl}$ at $37^{\circ} \mathrm{C}$ for $24 \mathrm{~h}$, followed by secondary enrichment in tryptic soy broth (Oxoid Ltd.) supplemented with $5 \mathrm{mg} / \mathrm{L}$ of oxacillin. After $24 \mathrm{~h}$ incubation at $37^{\circ} \mathrm{C}$, a loopful of enrichment broth was plated on Brilliance MRSA 2 agar (Oxoid Ltd.) and then incubated for further $24 \mathrm{~h}$. Presumptive MRSA colonies grown on Brilliance MRSA 2 agar were subcultured and confirmed by the above-cited multiplex PCR targeting the $S$. aureus-specific nuc gene and mecA gene. The MRSA strains were genotyped by multilocus sequence typing and by determination of sequence types, spa typing, SCCmec typing and subtyping, as previously described (Enright et al., 2000; Harmsen et al., 2003; Zhang et al., 2005; Kondo et al., 2007). The MRSA isolates were also tested for susceptibility to antimicrobials using the broth microdilution method, performed and interpreted according to the CLSI performance standard (CLSI, 2013). The following antimicrobials were tested: clindamycin, erythromycin, tiamulin, tetracycline, ciprofloxacin, rifampicin, cefoxitin, streptomycin, kanamycin, linezolid, fusidic acid, mupirocin, penicillin, vancomycin, sulphonamide, chloramphenicol, gentamycin, and trimethoprim. 
Staphylococcus aureus was detected in 85 out of 197 (43.1\%, 95\% CI: 36.2-50.1\%) bulk tank milk samples with counts ranging from 10 to more than $1.5 \times 10^{4}$ $\mathrm{cfu} / \mathrm{mL}$. This prevalence was similar to that reported in Switzerland by Muehlherr et al. (2003; 31.7\%), but different from that reported in other countries for this species: Chu et al. (2012) found a prevalence of $16.7 \%$ in Taiwan and Jørgensen et al. (2005) reported a prevalence of $96.2 \%$ in Norway. However, our prevalence was lower than that recently reported in Italy $(76.9 \%)$ by Spanu et al. (2013).

Methicillin-resistant $S$. aureus was found in 4 out of 197 bulk tank milk samples $(2.0 \%, 95 \%$ CI: $0.6-5.1 \%)$ : in 3 samples, the isolates were detected by direct plating, with the remaining 1 detected following enrichment. All 4 MRSA were mecA-positive isolates, and showed a phenotype of oxacillin-resistance. No mecCpositive isolate was detected in this study.

This is the first report on MRSA detection in goat milk in Italy and one of the few studies describing the presence of MRSA in goats. In particular, Statskova et al. (2009) isolated MRSA strains from individual and bulk milk samples and animal caretakers in Czech Republic. More recently, MRSA was detected in the nasal cavity, the vagina, and from individual dairy goat milk samples in Taiwan (Chu et al., 2012) and in milk samples obtained from clinical mastitis cases in Turkey (Aras et al., 2012), but the strains were not genotyped by multilocus sequence typing. Loncaric et al. (2013) isolated MRSA from the nasal cavity and reported lung infection in a goat, also suspecting a possible transmission of the same MRSA strain to farmers.

Three of our MRSA strains were classified as ST398, spa type t899, the dominant clone in livestock in Italy (Battisti et al., 2010; A. Battisti, IZSLT, Istituto Zooprofilattico Sperimentale delle Regioni Lazio e Toscana, IZSLT, Rome, Italy, personal communication), harboring a SCCmec type V cassette. The genotype of the remaining strain, isolated from the bulk tank milk of farm B, was ST1, spa type t127, and harbored a SCCmec type IVa (Table 1), a common feature among CC1 isolates of both human and animal origin. This clone was frequently reported in Italy in pigs, cattle, and humans, where it represents another prevalent lineage of LA-MRSA (Franco et al., 2011). Antimicrobial resistance patterns of the $4 \mathrm{MRSA}$ isolates are shown in Table 1. Isolates of CC398 from all the farms, in addition to $\beta$-lactams, were resistant to tetracycline, which characterizes almost all LA-MRSA clones circulating in Europe and Italy. Additionally, 2 of them were resistant to trimethoprim and 1 to clindamycin, common features among $S$. aureus isolates from livestock in Italy (Battisti et al., 2010; Feltrin et al., 2013). The resistance profile of the ST1 isolate was different, with additional resistances to aminoglycosides (kanamycin and streptomycin) and macrolides-lincosamides (erythromycin and clindamycin), a pattern previously reported in Italy (Franco et al., 2011).

Persistence of MRSA on the 4 MRSA-positive farms was investigated $1 \mathrm{yr}$ after the first examination. Three bulk tank milk samples retested positive for $S$. aureus, but only one (farm A) was still contaminated by MRSA (Table 1). In this farm, where goats were the only species reared, the half-udder milk from all lactating goats was collected and tested. Staphylococcus aureus was found in 16 out of 237 animals $(6.8 \%, 95 \%$ CI: $3.6-10.0 \%$ ), in 3 of which mecA-positive $S$. aureus (1.3\%, 95\% CI: $0.3-3.6 \%)$ were detected. In all 3 cases only one half-udder sample tested positive. In farm B, MRSA was not detected in either bulk tank milk, nor in the udder halves, although 5 goats tested positive for S. aureus $(4.9 \%, 95 \%$ CI: $0.7-9.1 \%)$. In both farms, no signs of clinical mastitis were observed in the goats. The absence of bulk tank milk contamination by MRSA in the subsequent year in 3 out of 4 farms could either be due to a clearance of the colonization, to the presence of only few goats excreting the pathogen in milk, or excreting it intermittently, or to the fact that some of the infected animals could have been removed during the time elapsed.

The same MRSA strains (i.e., same sequence type, spa type, SCCmec type and resistance profile), were isolated from the bulk tank milk on farm A in 2012 and 2013 and from the 3 infected goats. This provided evidence that the source of bulk milk contamination was very likely of mammary origin and that the presence of MRSA can persist for at least $1 \mathrm{yr}$ at the farm level. Methicillin-resistant S. aureus ST398 has been frequently described in cases of bovine mastitis (Fessler et al., 2010; Holmes and Zadoks, 2011), but this is the first time that this strain is found in goat bulk milk. In Italy, a case of serious infection due to MRSA ST398 spa type t899 was first described in a pig-farm worker (Pan et al., 2009), suggesting that people having close contact with MRSA ST398-shedding animals are at risk of being colonized by these strains. Unfortunately, we could not obtain samples from the personnel of farm A; however, during the study period, no clinical MRSA infection was reported among them.

In conclusion, high prevalence of $S$. aureus was detected in the bulk tank milk samples examined in this study. Because S. aureus is a frequent cause of foodborne disease, the application of stringent measures for the control of $S$. aureus mastitis in goat herds where unpasteurized milk intended for direct consumption or fresh cheese is produced seems appropriate to minimize the risk of food poisoning and colonization of occupationally exposed people. Further investigations 
are needed to genotype the isolates and determine the prevalence of enterotoxins and other virulence genes among $S$. aureus strains in our region.

The spread of antimicrobial resistant and multidrugresistant strains throughout the milk and dairy production chain is another matter of major concern. In this respect, the detection of MRSA in dairy goat milk, notwithstanding the low prevalence detected on the farms under study and at farm level, emphasizes the risks associated with the exposure of consumers and personnel to multidrug-resistant strains, which may cause colonization and disease. In this respect, goat bulk milk quality should be regularly monitored and microbiological investigations should be performed on a routine basis, including the screening of multidrug resistant and zoonotic pathogens.

\section{ACKNOWLEDGMENTS}

This work was funded by Italian Ministry of Health, Ricerca Corrente IZSLER 2010/03. The authors thank Francesca Pozzi (Istituto Zooprofilattico Sperimentale della Lombardia e dell'Emilia Romagna, Lodi, Italy) and Gessica Cordaro and Angela Ianzano (both of Istituto Zooprofilattico Sperimentale delle Regioni Lazio e Toscana, Rome, Italy) for their outstanding laboratory technical assistance.

\section{REFERENCES}

Aras, Z., I. Aydin, and K. Kav. 2012. Isolation of methicillinresistant Staphylococcus aureus from caprine mastitis cases. Small Rumin. Res. 102:68-73. http://dx.doi.org/10.1016/j. smallrumres.2011.08.014.

Battisti, A., A. Franco, G. Merialdi, H. Hasman, M. Iurescia, R. Lorenzetti, F. Feltrin, M. Zini, and F. M. Aarestrup. 2010. Heterogeneity among methicillin-resistant Staphylococcus aureus from Italian pig finishing holdings. Vet. Microbiol. 142:361-366. http:// dx.doi.org/10.1016/j.vetmic.2009.10.008.

Benedetti, V., P. Cremonesi, S. Ferrari, B. Castiglioni, M. Fabbi, N. Vicari, C. Garbarino, A. Battisti, A. Franco, F. Feltrin, and M. Luini. 2010. Staphylococcus aureus meticillino-resistenti (MRSA) da campioni di latte bovino. Large Anim. Rev. 16:67-70.

Bergonier, D., R. de Cremoux, R. Rupp, G. Lagriffoul, and X. Berthelot. 2003. Mastitis of dairy small ruminants. Vet. Res. 34:689-716. http://dx.doi.org/10.1051/vetres:2003030.

Chu, C., C. Yu, Y. Lee, and Y. Su. 2012. Genetically divergent methicillin-resistant Staphylococcus aureus and sec-dependent mastitis of dairy goats in Taiwan. BMC Vet. Res. 8:39. http://dx.doi. org/10.1186/1746-6148-8-39.

Clinical and Laboratory Standard Institute (CLSI). 2008. Performance Standards for Antimicrobial Disk and Dilution Susceptibility Tests for Bacteria Isolated From Animals; Approved Standard - Third Edition. CLSI Document M31-A3. CLSI, Wayne, PA.

Clinical and Laboratory Standards Institute (CLSI). 2013. Performance Standards for Antimicrobial Susceptibility Testing; Twenty-third Informational Supplement. CLSI Document M100-S23. CLSI, Wayne, PA.

Cremonesi, P., M. Luzzana, M. Brasca, S. Morandi, R. Lodi, C. Vimercati, D. Agnellini, G. Caramenti, P. Moroni, and B. Castiglioni. 2005. Development of a multiplex PCR assay for the identification of Staphylococcus aureus enterotoxigenic strains isolated from milk 
and dairy products. Mol. Cell. Probes 19:299-305. http://dx.doi. org/10.1016/j.mcp.2005.03.002.

Cuny, C., F. Layer, B. Strommenger, and W. Witte. 2011. Rare occurrence of methicillin-resistant Staphylococcus aureus CC130 with a novel mecA homologue in humans in Germany. PLoS ONE 6:e24360 http://dx.doi.org/10.1371/journal.pone.0024360.

Doyle, M. E., F. A. Hartmann, and A. C. Lee Wong. 2012. Methicillin-resistant staphylococci: implications for our food supply? Anim. Health Res. Rev. 13:157-180. http://dx.doi.org/10.1017/ S1466252312000187.

EFSA. 2009. Scientific Opinion of the Panel on Biological Hazards on a request from the European Commission on Assessment of the Public Health significance of methicillin resistant Staphylococcus aureus (MRSA) in animals and foods. EFSA J. 993:1-73.

Enright, M. C., N. P. Day, C. E. Davies, S. J. Peacock, and B. G. Spratt. 2000. Multilocus sequence typing for characterization of methicillin-resistant and methicillin-susceptible clones of Staphylococcus aureus. J. Clin. Microbiol. 38:1008-1015.

Feltrin, F., P. Alba, A. Ianzano, R. Amoruso, A. Caprioli, M. A. Argudín, B. Guerra, A. Battisti, and A. Franco. 2013. Molecular characterization of methicillin-resistant and methicillin-susceptible S. aureus (MRSA, MSSA) Clonal Complex 97 isolates from pigs and cattle, Italy. 3rd ASM-ESCMID Conference on Methicillinresistant Staphylococci in Animals: Veterinary and Public Health Implications. November 4-7, 2013, Copenhagen, Denmark.

Fessler, A., C. Scott, K. Kadlec, R. Ehricht, S. Monecke, and S. Schwarz. 2010. Characterization of methicillin-resistant Staphylococcus aureus ST398 from cases of bovine mastitis. J. Antimicrob. Chemother. 65:619-625. http://dx.doi.org/10.1093/jac/dkq021.

Foti, M., V. Fisichella, F. Conte, A. Passantino, and C. Giacopello. 2012. Indagine sulla presenza di Staphylococcus aureus meticillino resistenti (MRSA) in animali da reddito e in personale addetto agli allevamenti in Sicilia. Large Animal Rev. 18:177-182.

Franco, A., H. Hasman, M. Iurescia, R. Lorenzetti, M. Stegger, A. Pantosti, F. Feltrin, A. Ianzano, M. C. Porrero, M. Liapi, and A. Battisti. 2011. Molecular characterization of spa type t127, sequence type 1 methicillin-resistant Staphylococcus aureus from pigs. J. Antimicrob. Chemother. 66:1231-1235. http://dx.doi. org $/ 10.1093 / \mathrm{jac} / \mathrm{dkr} 115$.

Guardabassi, L., J. Larsen, J. S. Weese, P. Butaye, A. Battisti, J. Kluytmans, D. H. Lloyd, and R. L. Skov. 2013. Public health impact and antimicrobial selection of methicillin-resistant staphylococci in animals. J. Global Antimicrobial Resistance 1:55-62. http://dx.doi.org/10.1016/j.jgar.2013.03.011.

Harmsen, D., H. Claus, W. Witte, J. Rothgänger, H. Claus, D. Turnwald, and U. Vogel. 2003. Typing of methicillin-resistant Staphylococcus aureus in a university hospital setting by using novel software for spa repeat determination and database management. J. Clin. Microbiol. 41:5442-5448. http://dx.doi.org/10.1128/ JCM.41.12.5442-5448.2003.

Holmes, M. A., and R. N. Zadoks. 2011. Methicillin resistant S. aureus in human and bovine mastitis. J. Mammary Gland Biol. Neoplasia 16:373-382. http://dx.doi.org/10.1007/s10911-011-9237-x.

Jørgensen, H. J., T. Mørk, H. R. Høgåsen, and L. M. Rørvik. 2005. Enterotoxigenic Staphylococcus aureus in bulk milk in Norway. J.
Appl. Microbiol. 99:158-166. http://dx.doi.org/10.1111/j.13652672.2005.02569.x.

Kadariya, J., T. C. Smith, and D. Thapaliya. 2014. Staphylococcus aureus and staphylococcal food-borne disease: An ongoing challenge in public health. Biomed. Res. Int. http://dx.doi. org $/ 10.1155 / 2014 / 827965$.

Kondo, Y., T. Ito, X. X. Ma, S. Watanabe, B. N. Kreiswirth, J. Etienne, and K. Hiramatsu. 2007. Combination of multiplex PCRs for staphylococcal cassette chromosome mec type assignment: Rapid identification system for mec, $c c r$, and major differences in junkyard regions. Antimicrob. Agents Chemother. 51:264-274. http://dx.doi.org/10.1128/AAC.00165-06.

Loncaric, I., R. Brunthaler, and J. Spergser. 2013. Suspected goatto-human transmission of methicillin-resistant Staphylococcus aureus sequence type 398. J. Clin. Microbiol. 51:1625-1626. http:// dx.doi.org/10.1128/JCM.03052-12.

Muehlherr, J. E., C. Zweifel, S. Corti, J. E. Blanco, and R. Stephan. 2003. Microbiological quality of raw goat's and ewe's bulk-tank milk in Switzerland. J. Dairy Sci. 86:3849-3856. http://dx.doi. org/10.3168/jds.S0022-0302(03)73992-7.

Normanno, G., M. Corrente, G. La Salandra, A. Dambrosio, N. C. Quaglia, A. Parisi, G. Greco, A. L. Bellacicco, S. Virgilio, and G. V. Celano. 2007. Methicillin-resistant Staphylococcus aureus (MRSA) in foods of animal origin product in Italy. Int. J. Food Microbiol. 117:219-222. http://dx.doi.org/10.1016/j.ijfood micro.2007.04.006.

Pan, A., A. Battisti, A. Zoncada, F. Bernieri, M. Boldini, A. Franco, M. Giorgi, M. Iurescia, S. Lorenzotti, M. Martinotti, M. Monaci, and A. Pantosti. 2009. Community-acquired methicillin-resistant Staphylococcus aureus ST398 infection, Italy. Emerg. Infect. Dis. 15:845-847. http://dx.doi.org/10.3201/eid1505.081417.

Pantosti, A. 2012. Methicillin-resistant Staphylococcus aureus associated with animals and its relevance to human health. Front. Microbiol. 3:127. http://dx.doi.org/10.3389/fmicb.2012.00127.

Skov, R., A. R. Larsen, A. Kearns, M. Holmes, C. Teale, G. Edwards, and R. Hill. 2014. Phenotypic detection of mecC-MRSA: Cefoxitin is more reliable than oxacillin. J. Antimicrob. Chemother. 69:133-135. http://dx.doi.org/10.1093/jac/dkt341.

Spanu, V., C. Scarano, S. Virdis, S. Melito, C. Spanu, and E. De Santis. 2013. Population structure of Staphylococcus aureus isolated from bulk tank goat's milk. Foodborne Pathog. Dis. 10:310-315. http://dx.doi.org/10.1089/fpd.2012.1356.

Stastkova, Z., S. Karpiskova, and R. Karpiskova. 2009. Occurrence of methicillin-resistant strains of Staphylococcus aureus at goat breeding farm. Vet. Med. (Praha) 54:419-426.

Virdis, S., C. Scarano, F. Cossu, V. Spanu, C. Spanu, and E. De Santis. 2010. Antibiotic resistance in Staphylococcus aureus and coagulase negative staphylococci isolated from goats with subclinical mastitis. Vet. Med. Int. http://dx.doi.org/10.4061/2010/517060

Zhang, K., J. A. McClure, S. Elsayed, T. Louie, and J. M. Conly. 2005. Novel multiplex PCR assay for characterization and concomitant subtyping of staphylococcal cassette chromosome mec types I to $\mathrm{V}$ in methicillin-resistant Staphylococcus aureus. J. Clin. Microbiol. 43:5026-5033. http://dx.doi.org/10.1128/JCM.43.10.50265033.2005 . 